\title{
Spinal neuronal function reviewed after a motor complete spinal cord lesion
}

\author{
JJ Wyndaele \\ Antwerp University Hospital, Antwerp, Belgium \\ E-mail: spinalcord@uza.be
}

Dear Spinal Cord reader,

In this issue of Spinal Cord, several important studies and reviews are presented.

Hubli et al. describe the changes of spinal neuronal function that occur after a motor complete spinal cord injury (cSCI) in humans. Early after a cSCI, spinal reflex (SR) and locomotor activity are absent. After spinal shock, an early SR component reappears associated with the recovery of locomotor activity in response to appropriate peripheral afferent input. Clinical signs of spasticity take place in the following months, largely as a result of non-neuronal changes. After around 1 year the locomotor and SR activity undergo fundamental changes, i.e. the electromyographic amplitude in the leg muscles during assisted locomotion exhaust rapidly, accompanied by a shift from early to dominant late SR components. The exhaustion of locomotor activity is also observed in non-ambulatory patients with an incomplete SCI. At about 1 year after injury in most cSCI subjects the neuronal dysfunction is fully established and remains more or less stable in the following years. It is assumed that in chronic SCI the patient's immobility resulting in a reduced input from supraspinal and peripheral sources leads to a predominance of inhibitory drive within spinal neuronal circuitries underlying locomotor pattern and SR generation. The authors suggest that training of spinal interneuronal circuits including the enhancement of an appropriate afferent input might serve as an intervention to prevent neuronal dysfunction after SCI.

Curt et al. found that supernumerary phantom limbs were rather undocumented in traumatic SCI individuals. For appropriate diagnosis and treatment, supernumerary phantoms should be distinguished from other phantom sensations and pain syndromes.

DeVivo et al. provide guidelines for reporting results using the International Spinal Cord Injury Core Data Set. It is recommended that age at injury be reported by the mean, standard deviation, median, and range. When grouped, 15 year increments are recommended as follows: 0-15, 16-30, 31-45, 46-60, 61-75, 76+. For pediatric SCI, 0-5, 6-12, 13-15, 16-21 are recommended. Time since injury should be reported by mean, standard deviation, median, and range. The following intervals are recommended: $<1$ year, 1-5 years, 6-10 years, 11-15 years, and 5-year increments thereafter. Calendar time (years during which the study is conducted) is recommended grouped by either 5 or 10 year increments with years ending in 4 or 9. For 'Length of stay', the mean and standard deviation, as well as the median, are recommended for reporting. It is recommended that 'Severity of injury' is, under ordinary circumstances, reported in five categories: C1-4 ASIA Impairment Scale grade (AIS) A, B, or C; C5-8 AIS A, B, or C; T1-S5 AIS A, B, or C; AIS D at any injury level; and ventilator-dependent at any injury level or AIS grade. These recommendations should facilitate a more uniform reporting of the very basic core data on SCI and will facilitate comparison between different SCI studies.

Herrmann et al. validated the International Classification of Functioning, Disability and Health (ICF) Core Sets for individuals with SCI in the early post-acute and long-term context from the perspective of occupational therapists. Sixty seven experts from 27 countries addressed a vast variety of problems in their interventions in persons with SCI. The Comprehensive ICF Core Sets covered a high percentage of these problems. Further research will be necessary on a few aspects that are not included in the ICF Core Sets for SCI.

There is a much nice original research presented in this issue: Pouw et al. compared the neurological recovery and functional outcomes between traumatic central cord syndrome and motor incomplete tetraplegic individuals; Brown et al. compared exercise initiated in the acute and secondary phase of SCI in the rat; Lombardi et al. assessed the concomitant clinical improvement in different pelvic organ functions in incomplete spinal cord individuals using sacral neuromodulation; Saunders and Krause assessed risk factors with alcohol use in SCI individuals; Idowu et al. studied pressure ulceration in a resource-constrained setting; Bluvshtein et al. assessed insulin resistance in tetraplegia and paraplegia, and the role of the spinal cord in glucose regulation; Gélis et al. evaluated with a questionnaire the knowledge of self-reported pressure ulcer prevention measures; Chen et al. investigated the therapeutic effects of detrusor botulinum toxin A in individuals with different levels of SCI and types of detrusor sphincter dyssynergia. Hostettler et al. assessed the reliability of non-invasive cardiac output measurements using Innocor in individuals with tetraplegia at rest and during exercise and tested the hypothesis that CO-measurements are less reliable in these individuals.

Several Letters to the Editor are featured, related to the head-up tilt test and the 'Pain in your buttocks' manuscript, and a book review on neuroanatomy has been included.

Enjoy reading. 